\title{
Elasticity of a quantum monolayer solid
}

\section{Bruch, Ludwig Walter}

\section{Published in:}

Physical Review B

Link to article, DOI:

10.1103/PhysRevB.45.7161

Publication date:

1992

\section{Document Version}

Publisher's PDF, also known as Version of record

Link back to DTU Orbit

Citation (APA):

Bruch, L. W. (1992). Elasticity of a quantum monolayer solid. Physical Review B, 45(13), 7161-7164. https://doi.org/10.1103/PhysRevB.45.7161

\section{General rights}

Copyright and moral rights for the publications made accessible in the public portal are retained by the authors and/or other copyright owners and it is a condition of accessing publications that users recognise and abide by the legal requirements associated with these rights.

- Users may download and print one copy of any publication from the public portal for the purpose of private study or research.

- You may not further distribute the material or use it for any profit-making activity or commercial gain

- You may freely distribute the URL identifying the publication in the public portal

If you believe that this document breaches copyright please contact us providing details, and we will remove access to the work immediately and investigate your claim 


\title{
Elasticity of a quantum monolayer solid
}

\author{
L. W. Bruch \\ Department of Physics, University of Wisconsin-Madison, Madison, Wisconsin 53706 \\ and Fysisk-Kemisk Institut, The Technical University of Denmark, DTH 206, 2800 Lyngby, Denmark
}

(Received 12 November 1991)

\begin{abstract}
A perturbation-theory formulation of the zero-temperature elastic constants is used to verify symmetry relations for a (monolayer) triangluar lattice. A generalization of the Cauchy relation between the two elastic constants of the triangular lattice with central-pair-potential interactions is given for the quantum solid. The first-order quantum corrections are rederived in this formalism, and previous calculations are reanalyzed.
\end{abstract}

\section{INTRODUCTION}

The two-dimensional triangular lattice has an isotropic elasticity theory, ${ }^{1}$ in which the speeds of long-wavelength transverse and longitudinal sound waves are independent of the wave-vector direction. In the special case ${ }^{2}$ of a system governed by classical mechanics and with centralpair-potential interactions, there is a single independent elastic constant which relates the shear and bulk moduli. Here a perturbation theor $\mathrm{y}^{3,4}$ for the energy of distortions from the quantum ground state of the triangular lattice is used to explore relations among the elastic constants.

Macroscopic considerations ${ }^{1,2}$ on the harmonic deformation energy of a triangular lattice show that it can be expressed in terms of two parameters: the Lamé elastic constants which generate the bulk and shear moduli. If the undeformed lattice is under a static stress, ${ }^{5}$ the spreading pressure contributes to the effective elastic constants governing the speeds of sound. A static triangular lattice with pair potentials satisfies the conditions for a Cauchy solid, ${ }^{1,2}$ in which the two Lamé constants are equal. Indeed, in classical mechanics, it is quite straightforward to verify the isotropy condition and Cauchy relation from expressions which give the elastic constants as averages of interaction terms.

The purpose of this paper is to develop expressions ${ }^{4}$ for the zero-temperature elastic constants in terms of quantum-mechanical expectation values and thereby confirm or generalize relations which hold for the classical lattice. Previously, ${ }^{6}$ the shear elastic modulus was derived from the energy shift associated with a specific deformation, and the way in which various relations were fulfilled or failed was not clear. The work ${ }^{6}$ included Hartree and Jastrow variational approximations and a semiclassical approximation with strain derivatives of the normal-mode frequencies. ${ }^{7}$ Here the perturbation formulation of Feynman ${ }^{3}$ and McLellan ${ }^{4}$ is used to express the elastic constants in terms of the interactions and lattice sums, so that the way in which symmetry properties lead to relations among the elastic constants becomes clear. These results are used for a reevaluation of the semiclassical coefficients and for a reanalysis of the Jastrow varia- tional calculations. ${ }^{6}$

The organization of this paper is as follows: Section II contains the formulation of the elastic constants and symmetry arguments. Section III contains results of model calculations. Section IV contains concluding remarks.

\section{PERTURBATION THEORY OF THE ELASTIC CONSTANTS}

\section{A. Definitions}

The deformation tensor ${ }^{2,5,6} u_{i j}$ for a displacement of $\mathbf{x}$ to $\mathbf{x}^{\prime}$,

$$
\mathbf{x}^{\prime}=(\overleftrightarrow{\mathbb{1}}+\overleftrightarrow{\mathbf{u}}) \cdot \mathbf{x},
$$

is used to define the Lagrangian strain tensor

$$
\overleftrightarrow{\eta}=\frac{1}{2}\left(\overleftrightarrow{\mathrm{u}}+\overleftrightarrow{\mathrm{u}}^{T}+\frac{1}{2} \overleftrightarrow{\mathrm{u}}^{T} \overleftrightarrow{\mathrm{u}}\right) ;
$$

the isothermal elastic constants $C_{i j, k l}$ are coefficients in the quadratic form for the deformation free energy in terms of $\eta$. With a Voigt notation for the pairs of indices,

$$
1=x x, \quad 2=y y, \quad 3=x y=y x,
$$

the elastic constants discussed here are $C_{11}, C_{22}, C_{12}$, and $C_{33}$.

Macroscopic symmetries of the triangular lattice give

$$
\begin{aligned}
& C_{11}=C_{22}, \\
& C_{11}=C_{12}+2 C_{33} \quad \text { (isotropy). }
\end{aligned}
$$

The Lamé constants of isotropic elasticity theory are $\mathrm{a}^{1,2}$

$$
\lambda=C_{12}, \mu=C_{33},
$$

and a Cauchy solid is defined to have

$$
\mu-\lambda=0 \quad \text { (Cauchy) . }
$$

The bulk modulus for the triangular lattice is, in terms of the elastic constants,

$$
\begin{aligned}
B & =\left(C_{11}+C_{22}+2 C_{12}\right) / 4 \\
& =C_{12}+C_{33} \text { (isotropy); }
\end{aligned}
$$


it can also be obtained from density derivatives of the ground-state energy. When Eq. (2.7) holds, there is the remarkable situation that information about nontriangular, sheared, lattices is obtained from the bulk modulus for triangular lattices.

The shear modulus $C_{33}$ enters in the deformation energy for

$$
\overleftrightarrow{\mathrm{u}}\left(C_{33}\right)=(\widehat{\mathbf{x}} \widehat{\mathbf{y}}+\widehat{\mathbf{y}} \widehat{\mathbf{x}}) \delta .
$$

Calculations for a rectangular deformation at constant area, ${ }^{6}$

$$
\overleftrightarrow{\mathbf{u}}\left(C_{33}^{\prime}\right)=[\widehat{\mathbf{y}} \widehat{\mathbf{y}}-(1 / 1+\delta) \widehat{\mathbf{x}} \widehat{\mathbf{x}}] \delta,
$$

give an elastic modulus

$$
C_{33}^{\prime}=\frac{1}{4}\left(C_{11}+C_{22}\right)-\left(C_{12} / 2\right),
$$

which is equal to $C_{33}$ for the isotropic lattice. Calculations of the shear modulus using the deformation tensor of Eq. (2.10a) thus implicitly assumed that the lattice is isotropic. $^{6}$

In Sec. II C expressions for the elastic constants in terms of pair potentials and lattice sums are used to verify Eqs. (2.4) and (2.5) and to give the quantum generalization of Eq. (2.7).

\section{B. Perturbation theory \\ for the zero-temperature elastic constants}

McLellan ${ }^{4}$ formulated the elasticity theory of a quantum solid in terms of a Schrödinger perturbation theory with respect to the undeformed lattice. For a Hamiltonian which is a sum of kinetic and scalar potential energies,

$$
H_{0}=\frac{1}{2 m} \sum_{j} p_{j}^{2}+\Phi(\{\mathbf{r}\}),
$$

the perturbation operator is

$$
w(\{\mathbf{r}\})=H_{0}(\{(\overleftrightarrow{\mathbb{1}}+\overleftrightarrow{\mathbf{u}}) \mathbf{r}\})-H_{0}(\{\mathbf{r}\}) .
$$

Expressions for the elastic constants are obtained by collecting the contributions of first- and second-order perturbation theory which are quadratic in the Lagrange strain tensor. Here $\phi$ is assumed to be a sum of central pair potentials $\phi\left(r_{i j}\right)$ for $N$ atoms in an undeformed area $A$. The resolvent operator entering in the second-order perturbation theory is denoted

$$
R=\left(1-P_{0}\right) /\left(E_{0}-H_{0}\right),
$$

where $P_{0}$ is a projection operator for the ground state (of energy $E_{0}$ ) of the original Hamiltonian $H_{0}$.

Following McLellan, ${ }^{4}$ define a virial operator $V_{\alpha, \beta}$ by

$$
V_{\alpha, \beta}=-\frac{1}{m} \sum_{j} p_{j \alpha} p_{j \beta}+\sum_{i \neq j} \psi^{\prime \prime} r_{i j \alpha} r_{i j \beta},
$$

where the function $\psi$ is related to the pair potential $\phi$ by $\psi(x)=\phi(\sqrt{x})$, the prime denotes the derivative, $\alpha$ and $\beta$ denote Cartesian components, and the $i, j$ sums run over the $N$ atoms of the monatomic lattice. The stress tensor $S_{\alpha, \beta}$ is given by the ground-state expectation value of the virial operator

$$
S_{\alpha, \beta}=\left\langle V_{\alpha, \beta}\right\rangle_{0},
$$

and the virial equation of state for the spreading pressure is

$$
\pi A=-\left\langle V_{x x}+V_{y y}\right\rangle_{0} / 2
$$

The elastic constants are

$$
\begin{aligned}
& C_{11}=\frac{2}{A}\left(4\left\langle K_{x}\right\rangle_{0}+\sum_{i \neq j}\left\langle\psi^{\prime \prime} x_{i j}^{4}\right\rangle_{0}+\left\langle V_{x x} R V_{x x}\right\rangle_{0}\right), \\
& C_{12}=\frac{2}{A}\left(\sum_{i \neq j}\left\langle\psi^{\prime \prime} x_{i j}^{2} y_{i j}^{2}\right\rangle_{0}+\left\langle V_{x x} R V_{y y}\right\rangle_{0}\right) \\
& C_{33} \frac{2}{A}\left(\langle K\rangle_{0}+\sum_{i \neq j}\left\langle\psi^{\prime \prime} x_{i j}^{2} y_{i j}^{2}\right\rangle_{0}+\left\langle V_{x y} R V_{x y}\right\rangle_{0}\right)
\end{aligned}
$$

and

$$
\begin{aligned}
C_{33}^{\prime}=\frac{1}{2 A}[ & 4\langle K\rangle_{0}+\sum_{i \neq j}\left\langle\psi^{\prime \prime}\left(x_{i j}^{2}-y_{i j}^{2}\right)^{2}\right\rangle_{0} \\
& \left.+\left\langle\left(V_{x x}-V_{y y}\right) R\left(V_{x x}-V_{y y}\right)\right\rangle_{0}\right] .
\end{aligned}
$$

In Eq. (2.17) the notation $\left\langle K_{x}\right\rangle_{0}$ denotes the ground-state expectation value of the $x$ component of the total kinetic energy $K$. An expression for $C_{22}$ is obtained from Eq. (2.17) by replacing $x$ components by $y$ components. McLellan's expressions ${ }^{4}$ for the finite-temperature elastic constants contain fluctuation terms similar to those present in the classical statistical-mechanical theory. ${ }^{7}$

The bulk modulus is, from the first form of Eq. (2.8),

$$
\begin{aligned}
B=\frac{1}{2 A} & \left\langle 4\langle K\rangle_{0}+\sum_{i \neq j}\left\langle\psi^{\prime \prime} r_{i j}^{4}\right\rangle_{0}\right. \\
& \left.+\left\langle\left(V_{x x}+V_{y y}\right) R\left(V_{x x}+V_{y y}\right)\right\rangle_{0}\right) .
\end{aligned}
$$

Both the spreading pressure and bulk modulus can be calculated also from density derivatives of the ground-state energy.

\section{Symmetry relations}

The triangular lattice has sixfold rotation symmetry. Consequently, there are several relations among the ground-state expectation values in the expressions for the stress tensor and elastic constants in Sec. II B.

The stress tensor [Eq. (2.15)] is then diagonal and is related to the spreading pressure by

$$
S_{\alpha, \beta}=-\pi \delta_{\alpha, \beta} \text {. }
$$

The second-order perturbation terms in Eqs. (2.17)-(2.21) are related using the Wigner-Eckart Theorem, for matrix elements between states of specified total angular momentum and various space-axis projec- 
tions. Thus the isotropy relations Eqs. (2.4) and (2.5) are verified from the explicit expressions for the elastic constants, and the equivalence between $C_{33}$ and $C_{33}^{\prime}$ is shown.

However, the second-order terms do not cancel in the generalization of the Cauchy relation

$$
\begin{aligned}
\mu-\lambda & =C_{33}-C_{12} \\
& =\frac{2}{A}\left(\langle K\rangle_{0}+\left\langle V_{x y} R V_{x y}\right\rangle_{0}-\left\langle V_{x x} R V_{y y}\right\rangle_{0}\right),
\end{aligned}
$$

because they involve intermediate states of different angular momenta. The second-order terms reflect changes in the ground-state wave function; that they do not arise in the stress tensor is associated with the HellmanFeynman theorem for first derivatives of the ground-state energy with respect to external parameters. In classical statistical mechanics, the fluctuation terms ${ }^{7}$ in the elastic-constant theory also lead to a nonzero value of $\mu-\lambda$ for the central-pair-potential model at finite temperatures.

\section{MODEL CALCULATIONS}

The considerations by which the isotropy relations are derived from the Wigner-Eckart theorem, while the second-order terms do not cancel from the generalized Cauchy relation [Eq. (2.23)], are somewhat subtle. Therefore, in Sec. III A, results are presented for the first quantum corrections to the elastic constants, which show a nonvanishing remainder already in the leading order of quantum effects. Second, the perturbation expressions for the elastic constants consist of diagonal ground-state expectation values, which are readily calculated with variational trial functions, and more complex secondorder terms. A reanalysis of the semiclassical and Jastrow results ${ }^{6}$ is presented in Secs. III A and III B, separating the first- and second-order terms. The results of this section are for the Lennard-Jones $(12,6)$ pair potential

$$
\begin{aligned}
\phi(r) & =4 \epsilon\left[(\sigma / r)^{12}-(\sigma / r)^{6}\right] \\
& =\epsilon\left[\left(r_{0} / r\right)^{12}-\left(r_{0} / r\right)^{6}\right]
\end{aligned}
$$

and de Boer parameter

$$
\Lambda^{*}=h /(\sigma \sqrt{m \epsilon}) .
$$

Physical quantities are given in reduced form, with energies scaled by $\epsilon$ and elastic constants and spreading pressure scaled by $\epsilon / \sigma^{2}$.

The quantum hard-disk solid represents a singular case for the formulation of Sec. II. Values for the (pressure renormalized) Lamé constants of the hard-disk solid were obtained by Leung and Chester. ${ }^{8}$ Converting to the Lamé constants of this work shows that the interaction terms make a contribution to the right-hand side of Eq. (2.23), which is $\sim(-) 50 \%$ of the kinetic-energy term for the densities they treated.

\section{A. First-order quantum corrections to ground-state properties}

The first-order quantum corrections, linear in $\Lambda^{*}$, are calculated precisely, using quadratic expansions in normal-mode amplitudes for the operators in the formalism of Sec. II B. The first- and second-order perturbation terms both contribute to the first quantum correction. Table I contains results for the coefficient of $\Lambda^{*}$ in the spreading pressure, kinetic energy, and bulk and shear moduli. In addition, the coefficients of $\Lambda^{*}$ in the remainder

$$
\Delta^{*}=\mu^{*}-\lambda^{*}-\left(2\langle K\rangle_{0} \sigma^{2} / A \epsilon\right)
$$

and in the diagonal potential term of the bulk modulus

$$
B_{1}^{*}=\left(\sigma^{2} / 2 \epsilon A\right)\left\langle\sum_{i \neq j} \psi^{\prime \prime} r_{i j}^{4}\right\rangle_{0}
$$

are shown.

The range of lattice constants $L$ in Table I corresponds to values encountered for monolayer solids of the inertgas series from xenon to neon. Much of Table I represents a reevaluation of the coefficients which were obtained previously ${ }^{6}$ from strain derivatives ${ }^{7}$ of the normal-mode frequencies. The two calculations agree precisely, and the spreading-pressure and bulk-modulus coefficients are in good agreement with the results of finite-difference approximations to density derivatives of the zero-point energy.

There are terms zeroth order in $\Lambda^{*}$ in the spreadingpressure and elastic constants, available from lattice

TABLE I. First-order quantum corrections for the triangular lattice. ${ }^{\mathrm{a}}$

\begin{tabular}{lcccccc}
\hline \hline$L / r_{0}{ }^{\mathrm{b}}$ & $\pi^{*}$ & $\left(2\langle K\rangle_{0} / A\right)^{*}$ & $B^{*}$ & $C_{33}^{*}$ & $\Delta^{*}$ & $B_{1}^{*}$ \\
\hline 0.98 & 10.7 & 22.7 & 46.0 & 28.2 & 8.0 & 74.8 \\
1.00 & 9.0 & 17.9 & 37.5 & 23.6 & 7.9 & 64.7 \\
1.02 & 7.6 & 14.0 & 30.2 & 19.8 & 8.1 & 56.8 \\
1.04 & 6.6 & 10.8 & 23.2 & 16.6 & 8.7 & 50.9 \\
\hline \hline
\end{tabular}

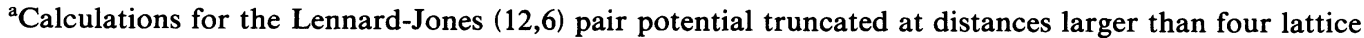
constants. Sums over wave vectors in a running-wave expansion of the perturbation-theory terms are done with a set of 45 special points (Ref. 6). Scalings $B_{1}^{*}$ and $\Delta^{*}$ are defined in Sec. III of the text. Entries are the scaled coefficients of the term first order in $\Lambda^{*}$.

${ }^{b}$ Nearest-neighbor distance (lattice constant) of the triangular lattice, scaled by the pair-potential minimum [Eq. (3.1)]. 
TABLE II. Jastrow variational calculations for the elastic moduli of monolayer helium solids. ${ }^{\text {a,b }}$

\begin{tabular}{cccccccr}
\hline \hline & $\rho^{*}$ & $\pi^{*}$ & $C_{33}^{*}$ & $C_{12}^{*}$ & $\mu^{*}-\lambda^{*}$ & $2 \rho^{*}\langle K\rangle / N \epsilon$ & $B_{1}^{*}$ \\
\hline \hline${ }^{3} \mathrm{He}$ & 0.51 & 0.59 & 2.36 & 0.43 & 1.94 & 1.23 & 9.01 \\
& 0.53 & 0.72 & 2.87 & 1.43 & 1.44 & 1.44 & 10.51 \\
& 0.55 & 0.91 & 3.53 & 2.66 & 0.87 & 1.58 & 15.51 \\
& 0.57 & 1.18 & 4.34 & 4.15 & 0.19 & 1.77 & 15.11 \\
& 0.59 & 1.51 & 5.25 & 5.90 & -0.65 & 1.98 & 18.33 \\
${ }^{3} \mathrm{He}$ & & & & & & & \\
& 0.51 & 1.05 & 3.31 & 0.64 & 2.67 & 1.57 & 11.42 \\
& 0.53 & 1.23 & 3.90 & 1.68 & 2.22 & 1.78 & 13.08 \\
& 0.55 & 1.48 & 4.74 & 2.95 & 1.79 & 2.01 & 15.28 \\
& 0.57 & 1.80 & 5.77 & 4.52 & 1.26 & 2.25 & 18.11 \\
& 0.59 & 2.20 & 6.98 & 6.43 & 0.55 & 2.51 & 21.65 \\
\hline \hline
\end{tabular}

${ }^{a}$ deBoer-Michels-Lennard-Jones (12,6) pair potential for helium; results taken from Ref. 6.

${ }^{b}$ Scaled density, spreading pressure, elastic constants, and kinetic-energy density, as in Sec. III of the text.

sums. However, for this (classical) limit, the remainder $\Delta^{*}$ and kinetic energy are zero. The spreading-pressure term is included because it enters in the effective elastic constants for the speeds of sound in the solid.

\section{B. Jastrow calculations for helium}

A reanalysis of the results of Jastrow calculations ${ }^{6}$ for the elastic constants of triangular lattices of helium is presented in Table II. The scaled number density is defined by $\rho^{*}=\rho \sigma^{2}$, and the range of densities there corresponds to much of the range ${ }^{9}$ for the adsorbed solids of helium isotopes on the basal-plane surface of graphite. The results are for the de Boer-Michels-Lennard-Jones potential $(\epsilon=10.22 \mathrm{~K}$ and $\sigma=2.556 \AA)$ with $\Lambda^{*}$ equal to 2.67 and 3.08 for ${ }^{4} \mathrm{He}$ and ${ }^{3} \mathrm{He}$, respectively. Note the contrasting trends with density of the remainder $\mu^{*}-\lambda^{*}$ in the Cauchy relation and of the kinetic-energy density. As in Table $I$, the first-order perturbation term $B_{1}^{*}$ is much larger than the net value $B^{*}$, showing the presence of a substantial (negative) second-order term.

\section{CONCLUDING REMARKS}

The perturbation-theory expressions for the elastic constants in terms of sums of interactions have the symmetries among the elastic constants of the triangular lattice, which are inferred from macroscopic considerations.

The remainder $\mu-\lambda$ in the quantum generalization of the Cauchy relation for the static triangular lattice with pair potentials involves more than the expectation value of the kinetic energy. Indeed, in variational calculations ${ }^{6}$ of the elastic constants, there were contributions from terms in the trial function which allowed for a change from triangular-lattice symmetry.

What are nominally first- and second-order perturbation contributions in the formal theory give comparable contributions to the quantum elastic constants.

\section{ACKNOWLEDGMENTS}

I thank Professor F. Y. Hansen and the Fysisk-Kemisk Institut for their hospitality during my visit at the Technical University of Denmark. The work was also supported in part by the National Science Foundation through Grant No. DMR-88-17761.
${ }^{1}$ G. A. Stewart, Phys. Rev. A 10, 671 (1974); J. Phys. (Paris) Colloq. 38, C4-207 (1977).

${ }^{2}$ D. C. Wallace, Thermodynamics of Crystals (Wiley, New York, 1972).

${ }^{3}$ R. P. Feynman, cited in J. M. Greif, Ph.D. thesis, California Institute of Technology, 1982.

${ }^{4}$ A. G. McLellan, J. Phys. C 17, 1 (1984); 17, 2999 (1984).

${ }^{5}$ T. H. K. Barron and M. L. Klein, Proc. Phys. Soc. London 85,
523 (1965)

${ }^{6}$ L. W. Bruch and J. M. Gottlieb, Phys. Rev. B 37, 4920 (1988).

${ }^{7}$ A. C. Holt, W. G. Hoover, S. G. Gray, and D. R. Shortle, Physica 49, 61 (1970); D. R. Squire, A. C. Holt, and W. G. Hoover, ibid. 42, 388 (1969).

${ }^{8}$ P. W. Leung and G. V. Chester, Phys. Rev. B 43, 735 (1991).

${ }^{9}$ J. M. Greif and D. L. Goodstein, J. Low Temp. Phys. 44, 347 (1981). 